\title{
Jet substructure shedding light on heavy Majorana neutrinos at the LHC
}

\section{Arindam Das}

School of Physics, KIAS, Seoul 02455, Korea E-mail: arindamekias.re.kr

\begin{abstract}
The existence of tiny neutrino masses and flavor mixings can be explained naturally in various seesaw models, many of which typically having additional Majorana type SM gauge singlet right handed neutrinos $(N)$. If they are at around the electroweak scale and furnished with sizable mixings with light active neutrinos, they can be produced at high energy colliders, such as the Large Hadron Collider (LHC). A characteristic signature would be same sign lepton pairs, violating lepton number, together with light jets $-p p \rightarrow N \ell^{ \pm}, N \rightarrow \ell^{ \pm} W^{\mp}, W^{\mp} \rightarrow j j$. We propose a new search strategy utilising jet substructure techniques, observing that for a heavy right handed neutrino mass $M_{N}$ much above $M_{W^{ \pm}}$, the two jets coming out of the boosted $W^{ \pm}$may be interpreted as a single fat-jet $(J)$. Hence, the distinguishing signal topology will be $\ell^{ \pm} \ell^{ \pm} J$. Performing a comprehensive study of the different signal regions along with complete background analysis, in tandem with detector level simulations, we compute statistical significance limits. We find that heavy neutrinos can be explored effectively for mass ranges $300 \mathrm{GeV} \leq M_{N} \leq 800 \mathrm{GeV}$ and different light-heavy neutrino mixing $\left|V_{\mu N}\right|^{2}$. At the $13 \mathrm{TeV}$ LHC with $3000 \mathrm{fb}^{-1}$ integrated luminosity one can competently explore mixing angles much below present LHC limits, and moreover exceed bounds from electroweak precision data.
\end{abstract}

39th International Conference on High Energy Physics

4-11 July 2018

Seoul, Korea 


\section{Introduction}

The experimental evidence for neutrino oscillations and lepton flavor mixings, from the various experiments, motivate extensions of the SM incorporating non-zero neutrino masses and mixings. After the pioneering realization of the unique $d=5$ Weinberg operator within the SM with $\Delta L=2$ lepton number violation ( $L=$ Lepton number), it was realized that the very well known Seesaw mechanism could be the simplest idea to explain the smallness of the neutrino masses and flavor mixings. In many of these models, SM is extended by gauge singlet, Majorana type, heavy right handed neutrinos (RHNs). After electroweak (EW) symmetry breaking, the light Majorana neutrino masses are generated by, for instance, the so called type-I seesaw mechanism. At the 8 TeV LHC, with $20.3 \mathrm{fb}^{-1}$ luminosity and $95 \%$ confidence limit (C. L.), ATLAS [1] has probed mixings for muon flavor down to a $\left|V_{\mu N}\right|^{2}$ of $3.5 \times 10^{-3}$, for $M_{N}=100 \mathrm{GeV}$. The limits further goes down to $2.9 \times 10^{-3}$ for $M_{N}=110 \mathrm{GeV}$ and then monotonically weakens with mass, up to $M_{N}=500 \mathrm{GeV}$. At $M_{N}=500 \mathrm{GeV}$ the limits are $\left|V_{\mu N}\right|^{2}=4 \times 10^{-1}$. The limits are nearly two orders of magnitude weaker in the case of electron flavor mixings $\left|V_{e N}\right|^{2}$ at the $95 \%$ C. L. CMS has also studied the SSDL plus dijet signal and obtain the exclusion limits for $\left|V_{e N}\right|^{2}$ [2] and $\left|V_{\mu N}\right|^{2}$ [3]. Both studies are performed at the $8 \mathrm{TeV}$ LHC with $19.7 \mathrm{fb}^{-1}$ luminosity at $95 \% \mathrm{C}$. L. The limits for the mixed $e^{ \pm} \mu^{ \pm}+j j$ final state was also considered in [2]. CMS observed upper limits for $\left|V_{e N}\right|^{2}$ at $1.2 \times 10^{-4}$ for $M_{N}=40 \mathrm{GeV}, 2 \times 10^{-2}$ for $M_{N}=85 \mathrm{GeV}, 8 \times 10^{-3}$ for $M_{N}=130 \mathrm{GeV}$ and $1.2 \times 10^{-2}$ for $M_{N}=200 \mathrm{GeV}$. Thus, the $\left|V_{e N}\right|^{2}$ limits were found to again weaken with $M_{N}$. Alternatively, RHNs may be excluded as large as $M_{N}=480 \mathrm{GeV}$, assuming the mixing is unity. The limits on $\left|V_{\mu N}\right|^{2}$ from the SSDL + dijet final state with $\mu$ flavor is probed down to $2 \times 10^{-5}$ for $M_{N}=40 \mathrm{GeV}, 4.5 \times 10^{-3}$ for $M_{N}=90 \mathrm{GeV}, 1.75 \times 10^{-3}$ for $M_{N}=125 \mathrm{GeV}$ and $7 \times 10^{-3}$ for $M_{N}=175 \mathrm{GeV}$ with $\left|V_{\mu N}\right|^{2}$ again weakening subsequently with $M_{N}$. For $M_{N}=500 \mathrm{GeV}$ the limit is $\left|V_{\mu N}\right|^{2}=0.6$. In this paper we leverage boosted $W^{ \pm}$production from massive RHN, and its subsequent decay into a fat-jet in association with $\mu^{ \pm} \mu^{ \pm}$pairs. The $P_{T}$ of the $W^{ \pm}$scale as $P_{T}^{W} \sim\left(M_{N}^{2}-M_{W}^{2}\right) / M_{N}$ and the separation between the hadronic decay products of $W^{ \pm}$scale as $\sim M_{W} / P_{T}^{W}$. Therefore, a natural region of focus may be the intermediate to heavy RHN mass range, say $M_{N} \geq 300 \mathrm{GeV}$. In this mass range, the only other competent limit that exists comes from indirect EW precision data (EWPD). The EWPD limit is around $\left|V_{\mu N}\right|^{2}=0.009[5,4,6]$. The boosted $W^{ \pm}$can produce a fat-jet when decays hadronically. For simplicity and clarity, we consider only the $\mu$ flavor for the SSDL. Moreover, $\mu$ detection efficiencies are better, compared to electrons and tau leptons. We place limits on $\left|V_{\mu N}\right|^{2}$ at the $13 \mathrm{TeV}$ LHC, with $3000 \mathrm{fb}^{-1}$ luminosity, in the $300 \mathrm{GeV} \leq M_{N} \leq 800 \mathrm{GeV}$ mass range which has been studied in [7].

\section{Collider Analysis}

To establish specific features and kinematic characteristics related to our RHN signal and backgrounds, we start by focusing on signal identification. Our prototypical signal is Same Flavour $\left(\mu^{ \pm}\right)$SSDL, in association with a fat-jet $(J)$. We adopt the selection criteria as :(i) muons $\mu^{ \pm}$are identified with a minimum transverse momentum $p_{T}^{\mu}>10 \mathrm{GeV}$ and rapidity range $\left|\eta^{\mu}\right|<2.4$, with a maximum efficiency of $95 \%$. Efficiency decreases for $p_{T}^{\mu}$ above $1 \mathrm{TeV}$, (ii) only events with reconstructed di-muons having same sign are selected for further analysis, (iii) hard jets having 


\begin{tabular}{|l||c|c|c||c|c|c|}
\hline \multicolumn{1}{|l||}{ Cut } & \multicolumn{3}{|c||}{ Signal for $M_{N}$} & \multicolumn{3}{c|}{ Background } \\
\hline \hline & $300 \mathrm{GeV}$ & $500 \mathrm{GeV}$ & $700 \mathrm{GeV}$ & $W W+j$ & $W Z+j$ & $W W Z+j$ \\
\hline Pre-selection + & & & & & & \\
$\mu^{ \pm} \mu^{ \pm}+J$ & $82.2+45.2$ & $36.6+23.4$ & $19.2+13.0$ & $2717.5+2597.0$ & $9881.3+7639.3$ & $252.1+240.4$ \\
$p_{T}^{J}>100 \mathrm{GeV}$ & {$[100 \%]$} & {$[100 \%]$} & {$[100 \%]$} & {$[100 \%]$} & {$[100 \%]$} & {$[100 \%]$} \\
\hline$p_{T}\left(\mu_{1,2}\right), m_{\mu \mu}$ & $79.5+39.8$ & $33.02+20.3$ & $15.6+9.2$ & $2255.7+2132.1$ & $5496.6+5074.1$ & $208.0+193.4$ \\
& {$[94 \%]$} & {$[88 \%]$} & {$[77 \%]$} & {$[83 \%]$} & {$[60 \%]$} & {$[82 \%]$} \\
\hline$E_{T}^{\text {miss }}<35 \mathrm{GeV}$ & $66.3+27.4$ & $28.5+18.1$ & $10.0+7.6$ & $260.8+163.2$ & $189.9+188.1$ & $24.2+19.6$ \\
& {$[74 \%]$} & {$[77 \%]$} & {$[55 \%]$} & {$[7.9 \%]$} & {$[2.2 \%]$} & {$[8.9 \%]$} \\
\hline$p_{T}^{J}>150 \mathrm{GeV}$ & $35.1+20.6$ & $15.2+10.5$ & $8.3+6.0$ & $152.4+91.4$ & $36.5+27.2$ & $14.14+12.4$ \\
& {$[44 \%]$} & {$[58 \%]$} & {$[44 \%]$} & {$[4.5 \%]$} & {$[0.4 \%]$} & {$[5.3 \%]$} \\
\hline$M_{J}>50 \mathrm{GeV}$ & $29.3+16.9$ & $20.9+10.2$ & $6.6+4.4$ & $34.0+26.6$ & $11.6+8.5$ & $6.6+5.0$ \\
& {$[36 \%]$} & {$[42 \%]$} & {$[34 \%]$} & {$[1.1 \%]$} & {$[0.1 \%]$} & {$[2.3 \%]$} \\
\hline$\tau_{21}^{\prime}<0.5$ & $26.7+13.7$ & $13.2+7.2$ & $5.4+2.8$ & $17.5+15.9$ & $5.9+5.2$ & $3.0+2.8$ \\
& {$[32 \%]$} & {$[34 \%]$} & {$[25 \%]$} & {$[0.6 \%]$} & {$[0.06 \%]$} & {$[1.2 \%]$} \\
\hline
\end{tabular}

Table 1: The effectiveness of different variables in minimizing backgrounds is illustrated in the form a cut flow. The two numbers correspond to expected events in $\mu^{+} \mu^{+}$and $\mu^{-} \mu^{-}$channels. We adopt a typical mixing angle $\left|V_{\mu N}\right|=0.03$. The numbers are for an integrated luminosity of $3000 \mathrm{fb}^{-1}$, at the $13 \mathrm{TeV} \mathrm{LHC}$.

at least $p_{T}^{j}>10 \mathrm{GeV}$ and $\left|\eta^{j}\right|<2.4$ are identified, (iv) candidate fat-jets are to be identified with $R=0.8$, Cambridge-Achen jet with $\left|\eta^{J}\right|<2.4$, (v) we identify the hardest fat-jet with the $W^{ \pm}$ candidate jet $(J)$, and this is required to have $p_{T}^{J}>100 \mathrm{GeV}$. These basic selection criteria are like primary level cuts required for effective signal identification. The last requirement is to ensure robust fat-jet properties. The features of the boosted fat-jet are rather more prominent for large $M_{N}$; showing up emphatically for $300 \mathrm{GeV}$ and above. Finally we introduce some additional event criteria and then illustrate various results by considering several signal benchmark points along with the SM backgrounds like $W W+j, W Z+j$ and $W W Z+j$. For final selection between the signal and background we list the final event selection criteria motivated by the kinematic distributions in [7]: (i) leading muon should have $p_{T}\left(\mu_{1}\right)>20 \mathrm{GeV}$ and the next hardest muon must have $p_{T}\left(\mu_{2}\right)>15 \mathrm{GeV}$, (ii) minimum invariant mass for the same sign muon pair must satisfy $m_{\mu \mu}>50 \mathrm{GeV}$. This is easily satisfied for the signal events, and can control backgrounds with non-prompt muon pairs, (iii)lacking any missing particles for our signal, require $E_{T}^{\text {miss }}<35 \mathrm{GeV}$. This can control background events with large MET contributions, (iv) the hardest, reconstructed fat-jet must have a transverse momentum $p_{T}^{J}>150 \mathrm{GeV}$, (v) we also demand the invariant mass of the hardest, reconstructed fat-jet to satisfy the jet mass $M_{J}>50 \mathrm{GeV}$. In principle one may use a mass window around the $W^{ \pm}$mass, but we find that a simple lower bound suffices. (vi) we use the $\mathrm{N}$-subjettiness ratio corresponding to the reconstructed fat-jet must satisfy $\tau_{21}^{J}<0.5$. The corresponding cut flow for the signal and SM backgrounds are listed in Tab. 1

\section{Conclusion}

We compare our results with the current LHC bounds from the $13 \mathrm{TeV}$ dilepton [9] and trilepton [10] searches and EWPD [5, 4, 6]. We find the RHNs can be probed up to 5- $\sigma$ significance at the $13 \mathrm{TeV}$ LHC using $3000 \mathrm{fb}^{-1}$ using SSDL plus fat-jet [7]. better than the EWPD. A comprehensive analysis using the opposite sign same flavor dilepton plus fat jet has been performed in [8].

\section{References}

[1] G. Aad et al. [ATLAS Collaboration], "Search for heavy Majorana neutrinos with the ATLAS detector in pp collisions at $\sqrt{s}=8$ TeV,' JHEP 1507, 162 (2015) doi:10.1007/JHEP07(2015)162 


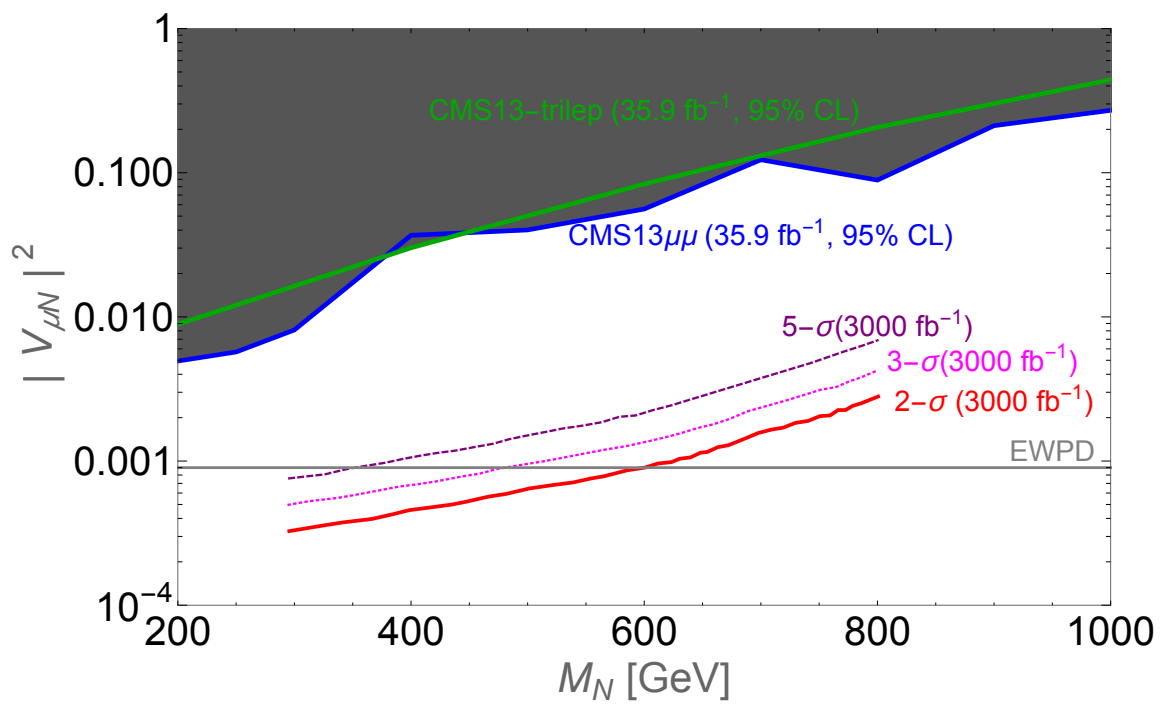

Figure 1: Comparison between current LHC limits ([9] and trilepton [10]) at the $13 \mathrm{TeV}$, EWPD [5, 4, 6] and calculated limits at the $13 \mathrm{TeV}$ LHC with $3000 \mathrm{fb}^{-1}$ luminosity at 2- $\sigma, 3-\sigma$ and $5-\sigma$ significances using the cut based analysis.

[arXiv:1506.06020 [hep-ex]].

[2] V. Khachatryan et. al. [CMS Collaboration], "Search for heavy Majorana neutrinos in $\mathrm{e}^{ \pm} \mathrm{e}^{ \pm}+\mathrm{jets}$ and $\mathrm{e}^{ \pm} \mu^{ \pm}+$jets events in proton-proton collisions at $\sqrt{s}=8 \mathrm{TeV}$," JHEP 1604, 169 (2016) doi: 10.1007/JHEP04 (2016)169 [arxiv:1603.02248 [hep-ex]].

[3] V. Khachatryan et al. [CMS Collaboration], "Search for heavy Majorana neutrinos in $\mu^{ \pm} \mu^{ \pm}+$jets events in proton-proton collisions at $\sqrt{s}=8 \mathrm{TeV}$," Phys. Lett. B 748, 144 (2015) doi:10.1016/j.physletb.2015.06.070 [arXiv:1501.05566 [hep-ex]].

[4] F. del Aguila, J. de Blas and M. Perez-Victoria, "Effects of new leptons in Electroweak Precision Data," Phys. Rev. D 78, 013010 (2008) doi:10.1103/PhysRevD.78.013010 [arXiv:0803.4008 [hep-ph]].

[5] J. de Blas, "Electroweak limits on physics beyond the Standard Model," EPJ Web Conf. 60, 19008 (2013) doi:10.1051/epjconf/20136019008 [arXiv:1307.6173 [hep-ph]].

[6] E. Akhmedov, A. Kartavtsev, M. Lindner, L. Michaels and J. Smirnov, "Improving Electro-Weak Fits with TeV-scale Sterile Neutrinos," JHEP 1305, 081 (2013) doi:10.1007/JHEP05(2013)081 [arXiv:1302.1872 [hep-ph]].

[7] A. Das, P. Konar and A. Thalapillil, "Jet substructure shedding light on heavy Majorana neutrinos at the LHC," JHEP 1802, 083 (2018) doi:10.1007/JHEP02(2018)083 [arXiv:1709.09712 [hep-ph]].

[8] A. Bhardwaj, A. Das, P. Konar and A. Thalapillil, "Looking for Minimal Inverse Seesaw scenarios at the LHC with Jet Substructure Techniques," arXiv:1801.00797 [hep-ph].

[9] A. M. Sirunyan et al. [CMS Collaboration], "Search for heavy Majorana neutrinos in same-sign dilepton channels in proton-proton collisions at $\sqrt{s}=13 \mathrm{TeV}$," arXiv:1806.10905 [hep-ex].

[10] A. M. Sirunyan et al. [CMS Collaboration], "Search for heavy neutral leptons in events with three charged leptons in proton-proton collisions at $\sqrt{s}=13 \mathrm{TeV}$," Phys. Rev. Lett. 120, no. 22, 221801 (2018) doi:10.1103/PhysRevLett.120.221801 [arXiv:1802.02965 [hep-ex]]. 